\title{
Pilot study of Internet-based early intervention for combat-related mental distress
}

\author{
Benjamin W. Van Voorhees, MD, MPH; ${ }^{\mathbf{*}}{\text { Jackie Gollan, PhD; }{ }^{2} \text { Joshua Fogel, } \mathbf{P h D}}^{\mathbf{3}}$ \\ ${ }^{1}$ RISE Consulting, Flossmoor, IL; General Pediatrics and Adolescent Medicine, Children's Hospital University of Illi- \\ nois, Chicago, IL; and University of Illinois at Chicago College of Medicine, Chicago, IL; ${ }^{2}$ Department of Psychiatry \\ and Behavioral Sciences, Northwestern University, Chicago, IL; ${ }^{3}$ Department of Finance and Business Management, \\ Brooklyn College, City University of New York, Brooklyn, NY
}

\begin{abstract}
This article evaluates an Internet-based early intervention combining online cognitive-behavioral therapy (CBT) with electronic peer-to-peer support intended to promote mental health and well-being among combat veterans. We conducted a phase 1 clinical trial of 50 Iraq and Afghanistan veterans using a pre and post single-arm design. We evaluated feasibility and changes in mental health symptoms (depression and posttraumatic stress disorder [PTSD]), functional status, and attitudes toward treatment seeking at baseline and weeks 4 , 8 , and 12 . A diverse group of veterans was enrolled (26\% ethnic minority, $90 \%$ male, $66 \%$ with income $<\$ 30,000 /$ year, $88 \%$ with no prior treatment for depression). Participants completed a mean of 4 of 6 lessons (standard deviation $=2.54$ ). From baseline to week 12, there were significant declines in the Center for Epidemiologic Studies-Depression scale score (effect size $[\mathrm{ES}]=0.41)$ and PTSD Checklist-Military version score $(E S=0.53)$. There were significant improvements in willingness to accept diagnosis $(E S=1.08)$ and perceived social norms and stigma regarding friends $(\mathrm{ES}=1.51)$. Although lack of a control group is a limitation, the Internet-based program combining CBT-based coping skills training and peer-topeer support demonstrated potential feasibility and evidenced benefit in symptom remediation for depression and PTSD.
\end{abstract}

Clinical Trial Registration: ClinicalTrials.gov; NCT01231711, "Improving Quality-of-life and Depressive Symptoms of Combat Veterans Via Internet-based Interventions”;

http://www.clinicaltrials.gov.
Key words: cognitive therapy, combat disorders, depressive disorder, early intervention (education), Internet, military personnel, posttraumatic, prevention and control, stress disorders, veteran health, veterans.

\section{INTRODUCTION}

Returning servicemembers experience a range of mental disorders and symptoms. Most prominent are posttraumatic stress disorder (PTSD; 21.8\% Department of Veterans Affairs [VA] sample, 4.8\% returning servicemembers sample) and depression (17.4\% VA sample, $11.0 \%$ returning

\footnotetext{
Abbreviations: $\mathrm{ANOVA}=$ analysis of variance, $\mathrm{BA}=$ behavioral activation, $\mathrm{CBT}=$ cognitive-behavioral therapy, CES-D = Center for Epidemiologic Studies-Depression, CI = confidence interval, $\mathrm{ES}=$ effect size, $\mathrm{IM}=$ instant messaging, $\mathrm{MI}=$ motivational interview, $\mathrm{OEF}=$ Operation Enduring Freedom, OIF = Operation Iraqi Freedom, PCL-M = PTSD Checklist-Military version, PST $=$ problem-solving therapy, PTSD = posttraumatic stress disorder, SD = standard deviation, SF-12 = 12-Item Short Form Health Survey, TPB = Theory of Planned Behavior, VA = Department of Veterans Affairs.

*Address all correspondence to Benjamin W. Van Voorhees, MD, MPH; RISE Consulting, 845 Bruce Ave, Flossmoor, IL 60422; 708-704-8846. Email: bvanvoor@uic.edu http://dx.doi.org/10.1682/JRRD.2011.05.0095
} 
servicemembers sample) [1-2]. Of servicemembers who met the criteria for one of these disorders, only 38 to 45 percent indicated an interest in receiving help; furthermore, only 23 to 40 percent reported actually receiving professional help within the previous year [1,3]. A combination of mental disorder symptoms, failure to initiate an effective intervention directed by self or behavioral health professionals, and preexisting educational and situational disadvantages increases the risk of unsuccessful transition to civilian life or impairment of military roles [4-5].

Complex and multidimensional pathways have been linked with risk for PTSD and depression among servicemembers exposed to combat and operational trauma. Though each disorder has specific theorized pathways to onset, PTSD and depressive disorder share common factors thought to increase vulnerability in three domains: behavioral (avoidance), cognition (learning and informationprocessing bias, low problem-solving orientation), and interpersonal relations (low levels of social support). Conversely, several common resiliency factors have been identified for these disorders in the same domains that are expected to be protective based on prior studies of mental disorders: behavioral (engagement in meaningful activities), cognitive (self-efficacy, problem-solving skills), and interpersonal relations (enhanced social support) [6-7]. However, negative affect can exacerbate both disorders, increasing functional impairment and worsening corespecific symptoms [8-11]. Without intervention, both disorders impair the individual's performance in work, military, and personal roles [5].

Our rationale for developing a new veteran-focused, Internet-based intervention is threefold: (1) the availability of common vulnerability and protective factors for intervention (see previous paragraph), (2) the need to address multiple barriers to early intervention, and (3) empirical evidence supporting common psychotherapeutic approaches. With regard to the second rationale, treatment seeking in both civilian and servicemember populations is impaired by the system (geographic access), the provider (insufficient supply, lack of training), the community (negative social norms/stigma), and individual factors (unfavorable attitudes toward interventions) [12]. It is unrealistic to assume that veterans will seek treatment for specific psychological disorders given that most individuals have difficulty "constructing" the concept of common mental disorder [13]. Internet-based interventions can address all these barriers because they are universally distributed; available at any time; and can be delivered privately to avoid stigma, enhance self-efficacy, and minimize negative attitudes toward mental health interventions [14-15]. With regard to the third rationale, cognitive-behavioral therapy (CBT) strategies delivered online are known to be an efficient, effective, and low-cost option for reducing preclinical distress and impairment and mitigating symptoms of deployment-related mental health disorders, including depression and PTSD [16-17].

We responded to this need by developing VETS PREVAIL, a transdiagnostic (addressing symptoms across several diagnostic categories, e.g., depression and anxiety), Internet-only early intervention that includes structured learning of coping skills related to common pathways of illness and impairment. Users are supported by peer-to-peer chats and social worker-provided motivational interview (MI) techniques. The intervention enhances the three protective domains of functioning for PTSD and depression and is an early intervention in the Institute of Medicine model, providing indicated prevention for servicemembers with subthreshold PTSD and depression symptoms and case identification treatment for those above the threshold [14]. We conducted a pre/post design phase 1 pilot study with 50 veterans to examine feasibility (adherence and sociocultural relevance and satisfaction), clinical response (change in depressed mood, PTSD symptoms, and functional status), and change in attitudes relevant to treatment seeking (change in intention, attitudes and beliefs toward intervention, social norms, and self-efficacy). We hypothesized that at least half the participants would complete the intervention and that they would report favorable ratings in sociocultural relevance (hypothesis 1) and that favorable changes would be seen in mental disorder symptoms of depression and PTSD (hypothesis 2), functional status (hypothesis 3), and attitudes toward mental health care seeking (hypothesis 4).

\section{METHODS}

\section{Study Design}

Working as an independent consultant (RISE Consulting) to Prevail Health Solutions, Dr. Van Voorhees supervised a phase 1 pilot study of the potential feasibility, benefit, and safety of the VETS PREVAIL intervention. The overall study design was a single-group, pre/post comparison (screening and baseline compared with follow-up at weeks 4, 8, and 12). Recruitment began on September 19, 2009, and continued until November 11, 2009, during 
which we enrolled the planned cohort of 50 participants. Data collection continued until April 15, 2010. Participants were recent U.S. veterans from any military branch (deployed after September 11, 2001) of Operation Iraqi Freedom (OIF) and Operation Enduring Freedom (OEF) who were experiencing depression and/or distress symptoms at the time of screening (Center for Epidemiologic Studies-Depression [CES-D] 20 score $>8$ ) but were not considered to be too severely ill for an early intervention (CES-D 20 score $>35$, indicating severe depressed mood or exhibiting self-harm risk).

\section{Intervention Contents}

The VETS PREVAIL intervention encompasses five online elements thought to promote behavior change for mental health problems within a framework of "behavioral vaccines” that include (1) effective components (1 element), (2) a motivational framework (2 elements), and (3) an implementation structure (2 elements) [18].

\section{Effective Components}

The effective components include structured lessons with curriculum reflecting the evidence-based current situation-oriented approaches of behavioral activation (BA) and problem-solving therapy (PST) (element 1) [19-20]. Element 1 is an online series of six half-hour eLearning "lessons" that convey CBT concepts, with an emphasis on BA and PST with the perspective that current situation-oriented approaches would be most easily accepted and relevant to returning combat veterans (full description provided in Table 1).

\section{Motivational Framework}

The motivational framework comprises the elements of engagement and guidance by a professional social worker to enhance adherence (element 2) [21] and the opportunity to interact with peers to relate learned materials to participants' current concerns (element 3) [22-23]. With regard to element 2, a master's level social worker reviewed progress and conducted brief weekly MIs with participants via instant messaging (IM) chats to sustain adherence. In terms of element 3, VA Certified Peer Counselors conducted a series of brief peer-to-peer IM chats to establish personal relevance and ensure completion of CBT content and tasks (e.g., compliance with BA). Both professional guidance (element 2) and peer support (element 3) are modeled after MI techniques, providing a framework of engagement [24-25].

\section{Implementation Structure}

Regarding implementation structure, the intervention includes cultural adaptation to the needs of servicemembers and veterans (element 4) [3,26] and tailoring to specific demographic and psychological needs (element 5) [27]. With regard to element 4, lack of culturally appropriate materials can be a major impediment to using behaviorchange interventions in mental health [28]. Materials were carefully developed using focus group direct input from veterans to ensure that the situational learning that is the foundation of the program was realistic and relevant (see Figures 1 and 2 for examples). In terms of element 5, a

Table 1.

Description of online structured learning lessons in VETS PREVAIL intervention.

No. Lesson

1 Discusses normal, subsyndromal responses to combat and operational stress; introduces symptoms of PTSD and depression; lays foundation for teaching about common protective factors that can promote resilience against these disorders.

2 Introduces concept of avoidance as coping mechanism, including procrastination, spacing out, and overthinking; teaches how these behaviors can worsen symptoms of PTSD and depression; illustrates how established routines and activities can reduce symptoms.

3 Teaches concept of triggers that can lead to dysfunctional thoughts; encourages users to re-evaluate these thoughts and plan adaptive responses.

4 Takes users through process of defining problem, brainstorming solutions, employing decision-making strategy, and arriving at realistic action plan.

5 Focuses on importance of setting goals; teaches about difference between helpful and unhelpful goals.

6 Introduces concept that taking time to experience environments closely can bolster resilience against mental distress; recaps all concepts covered in earlier lessons. 


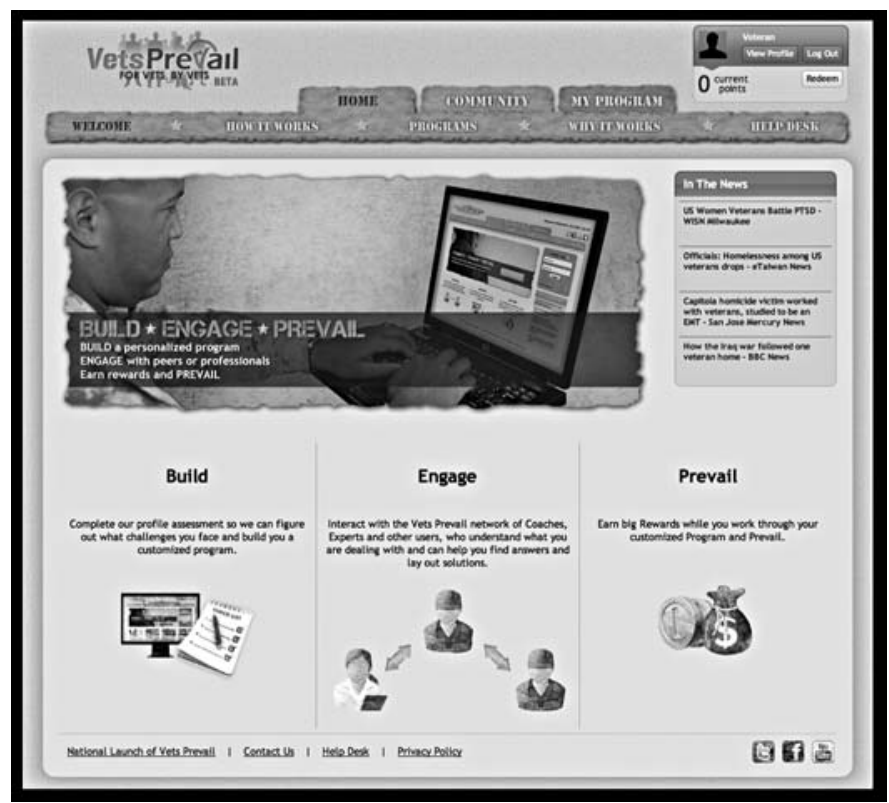

Figure 1.

VETS PREVAIL intervention homepage.

rule-based system was combined with an expansive library of multimedia "assets" to tailor delivery of content to the unique psychological needs and demographic characteristics of the individual veteran participant [29].

\section{Recruitment of Participants}

We recruited potential participants by placing announcements on the Prevail Health Solutions Web site, social media sites (Facebook and Twitter), and Craigslist. ${ }^{*}$ We asked those interested in the study to register online, complete a three-stage screener (including a self-report of clinical measures and a telephone call to research study staff), and sign an electronic consent form in order to be enrolled. Following completion of the clinical measures, we asked the eligible participants to contact a veteran staff member by telephone to verify their veteran status. An example of the questions asked to verify the status included: "When were you deployed in support of OIF and OEF, and to where?" Once their veteran status was verified, the potential participants were consented and

\footnotetext{
*"Craigslist is a centralized network of online communities featuring free online classified advertisements, with sections devoted to jobs, housing, personals, for sale, services, community, gigs, résumés, and discussion forums.” (Source: http://en.wikipedia.org/wiki/Craigslist)
}

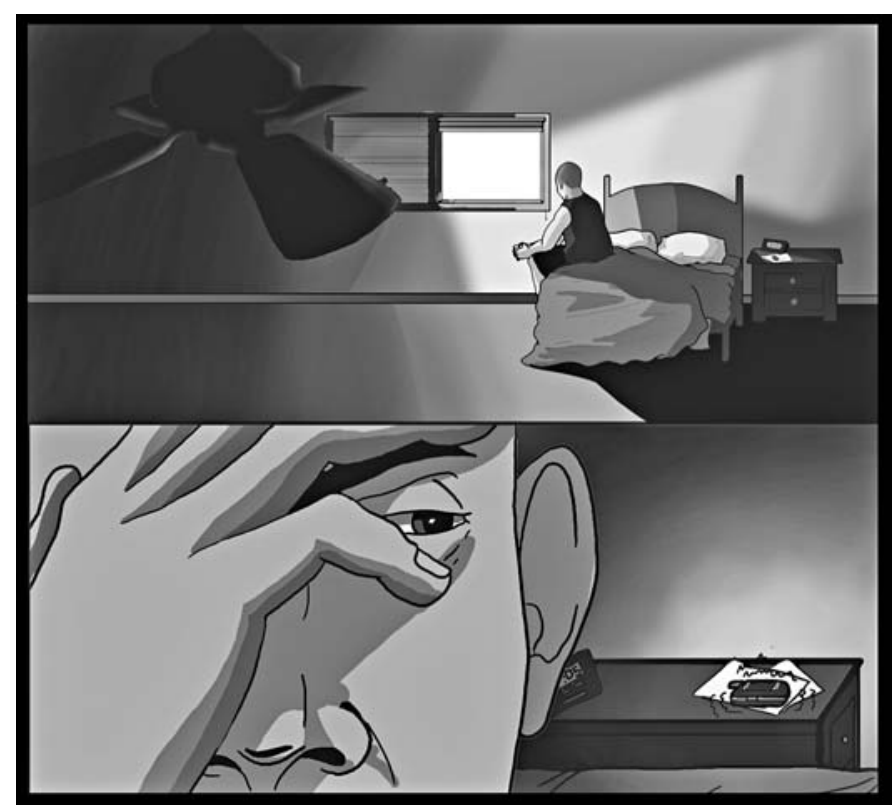

Figure 2.

Screenshot from animated sequence in VETS PREVAIL intervention.

asked to make an appointment for an orientation telephone call to help them start the online study.

\section{Inclusion and Exclusion Criteria}

Eligible participants were aged $\geq 18$ yr and veterans having served in OIF and/or OEF in the last 5 yr. We excluded those with no access to the Internet, no cellular telephone, or no cellular text-messaging capability. We also excluded those considered to be at high risk for suicide attempts: (1) psychiatric hospitalization within the past $10 \mathrm{yr}$, (2) past suicide attempt and/or diagnosis of schizophrenia or bipolar disorder, (3) discontinued or altered dosage of medication(s) within $10 \mathrm{~d}$ prior to enrollment, or (4) CES-D 20 score $>35$. We also excluded those insufficiently symptomatic to engage the material (CES-D 20 score $<9$, mean for general population) [30-31]. We assessed past suicide attempts, psychiatric hospitalizations, diagnoses of schizophrenia or bipolar disorder, and medication usage via self-report on the Personal Information Questionnaire.

\section{Informed Consent}

Participants read and electronically signed a printable consent form before any participation began and had elements of consent confirmed by telephone interview with 
study personnel. Participants who were unable to print a copy could request that a copy of the consent form be sent via postal mail. The consent form advised participants to continue any mental health therapy in which they were currently enrolled. They were told that the study was not intended to replace their existent care arrangements, which could include face-to-face therapy, group therapy, or drug therapy. They were also informed of the mental health services available to OIF/OEF veterans at the Chicago-area Jesse Brown VA Medical Center or national help lines for those outside of the Chicago area.

\section{Compensation of Participants}

All participants received a total of \$150 in compensation for completing four sessions of self-rating scales at baseline and weeks 4,8 , and 12 . Participants received the first payment of $\$ 20$ after completing the initial session of self-rating scales at baseline.

\section{Protection of Participants}

We employed a protocol for evaluating self-harm risk using established instruments and responding based on level of risk. A licensed clinical psychologist was continuously on call during the study to ensure adherence to this protocol and respond to any report of self-harm ideation.

\section{Data Collection}

We obtained all outcomes through the Internet using self-report questionnaires completed at baseline and weeks 4,8 , and 12. We monitored use of the intervention Web site, recording for each participant time spent on the Web site, number of logins, and number of characters typed. We also recorded all electronic communications sent to and from participants. The principal investigator monitored communications to assess the peers' and social workers' adherence to protocol and also provided any necessary feedback to ensure protocol adherence.

\section{Study Measures}

\section{Demographic Characteristics}

We asked participants to report their current age, sex, race/ethnicity, and branch of service. With regard to socioeconomic status, we asked for the years of education and also highest level of education achieved (high school/GED [general equivalency diploma], vocational, associate's degree, bachelor's degree, graduate degree), marital status (single, married, divorced, widowed), whether they had children, total yearly household income by categories $(<\$ 10,000, \$ 10,000-\$ 19,999$, etc.), and employment status (student working, student not working, employed part-time, employed full-time, not a student/not employed). In terms of history of mental disorders, we asked: "Has anyone in your family ever been diagnosed with depression?” (yes, no, and don't know), "Have you ever had a prior diagnosis of depression?" (yes, no), and if yes, "How was your depression treated?” (not applicable, no treatment, counseling only, counseling and medication, medication only).

\section{Feasibility}

We assessed feasibility by measuring adherence to and sociocultural relevance and satisfaction with the program. With regard to adherence, we report on the number of elearning lessons completed, the number of chats completed, and total number of characters typed in the chats [10]. We asked each participant to complete six e-lessons, which were tracked by the research team for completion (the lessons were interactive and required participants to periodically click various elements to advance; progress through the lessons was tracked by recording these clicks). We considered each lesson complete only after the participant clicked "Finish" at the end of that lesson. We did not record the time spent on each lesson. We told the participants there were two different types of chats: chats prompted before completing a lesson and chats prompted after completing a lesson. The chats prompted before the lesson were between the participant and a veteran peer (member of the research team) and primarily focused on building rapport through discussion of shared military experience. The chats prompted after a lesson were between the participant and a master's level social worker (member of the research team) and focused on discussing that lesson's content. In addition, participants could log onto the study Web site and initiate a chat with a veteran peer at any time. Several veteran peers and social workers conducted the chats, and participants were not necessarily connected with the same veteran or social worker every chat. We did not record the length of each chat. We based the user satisfaction domains on assessments piloted and used in a phase 2 randomized clinical trial $[6,10]$. Veteran participants rated sociocultural relevance (5-point Likert scale, where $1=$ strongly disagree and $5=$ strongly agree) across three separate domains: (1) overall satisfaction (e.g., "I would recommend VETS PREVAIL to a friend dealing with combat or postdeployment stress”), (2) helpfulness 
(e.g., "I received practical advice about how I can handle my emotions"), and (3) personal relevance (e.g., "The VETS PREVAIL training program struck a chord with my own life"). Table 2 provides the full list of statements in each domain.

\section{Symptoms}

We assessed clinical outcomes in three key domains of servicemember health using widely accepted instruments with established validity and reliability: (1) depressed mood and general distress, (2) PTSD symptoms, and (3) functional status. We measured depressed mood using the CES-D 10 (a subset of the CES-D 20) [31] at time of screening; baseline; and weeks 4,8 , and 12 . The CES-D 10 is a 10 -item questionnaire used to assess depressed mood scored from 0 to 30 based on frequency of symptoms during the previous week $(0=$ not at all to $3=$ nearly every day; Cronbach alpha = $0.66 / 0.76$ ) [31-32]. We also report those with clinically significant symptoms of depression (CES-D 10 score $>9$ ) at screening and most recent follow-up. We measured PTSD symptoms using the PTSD Checklist-Military version (PCL-M) at baseline and weeks 4, 8, and 12. The PCL-M is a 17-item self-report measure of the 17 symptoms of PTSD defined in the Diagnostic and Statistical Manual of Mental Disorders, 4th edition. Respondents rate how much they were "bothered by [problem] in the past month." Items are rated on a 5-point Likert scale, where $1=$ not at all and $5=$ extremely, for a range of scores from 17 to 85 . The PCL-M asks about problems in response to "stressful military experiences" and has demonstrated satisfactory reliability and validity (Cronbach alpha $=0.91 / 0.93)$ [30]. We also report those with clinically significant symptoms of posttraumatic stress (PCL-M score $>30$ ) at baseline and most recent follow-up [30].

\section{Functional Status}

We measured functional status using the 12-Item Short Form Health Survey (SF-12) mental and physical component scores at baseline and weeks 4, 8, and 12 [9]. The SF-12 is a health questionnaire that examines healthrelated quality of life across both physical (Cronbach alpha $=0.66 / 0.80)$ and mental (Cronbach alpha $=0.72 /$ $0.80)$ domains [33].

Attitudes Toward Treatment Seeking, Mental Health SelfEfficacy, and Stigma

We selected items to evaluate attitudes toward mental health treatment based on the framework of the Theory of Planned Behavior (TPB), which provides a framework for understanding intention to seek treatment. We previously demonstrated associations between items in this framework and intention to seek treatment or actual treatment seeking [13,34-36]. The TPB states that intention immediately precedes action and is directly influenced by attitudes and beliefs toward a behavior (e.g., attitudes toward intervention), subjective norms (e.g., concerns with regard to

Table 2.

Sociocultural relevance and satisfaction $(n=31)$.

\begin{tabular}{|c|c|c|c|c|}
\hline Item $^{*}$ & $\begin{array}{l}\text { All Responses } \\
(\text { mean } \pm \text { SD) }\end{array}$ & $\begin{array}{l}\text { Satisfied, } \\
\text { n (\%) }\end{array}$ & $\begin{array}{l}\text { Not Satisfied, } \\
\quad n(\%)\end{array}$ & $\begin{array}{l}\text { Neither, } \\
n(\%)\end{array}$ \\
\hline \multicolumn{5}{|l|}{ Satisfaction } \\
\hline Rate your overall satisfaction with VETS PREVAIL program. & $4.29 \pm 0.90$ & $27(87.10)$ & $1(3.23)$ & $3(9.68)$ \\
\hline $\begin{array}{l}\text { I would recommend VETS PREVAIL to friend dealing with combat } \\
\text { or postdeployment stress. }\end{array}$ & $4.52 \pm 0.89$ & 28 (90.32) & $1(3.23)$ & $2(6.45)$ \\
\hline I received helpful information and advice about stress and depression. & $4.23 \pm 0.88$ & $27(87.10)$ & $1(3.23)$ & $3(9.68)$ \\
\hline $\begin{array}{l}\text { I feel more in control of my life after going through VETS PREVAIL } \\
\text { program. }\end{array}$ & $3.81 \pm 0.83$ & $19(61.29)$ & $1(3.23)$ & $11(35.48)$ \\
\hline I received practical advice about how I can handle my emotions. & $4.13 \pm 0.92$ & $25(80.65)$ & $1(3.23)$ & $5(16.13)$ \\
\hline
\end{tabular}


family, peer, or employer opinions), and perceived behavioral control (e.g., self-efficacy), affecting the probability that one will perform the specific behavior (e.g., adhering to the intervention) [37]. Participants rated agreement (5-point Likert scale, where 1 = strongly disagree and 5 = strongly agree) with items selected based on our prior work on attitudes and intent $[13,36]$. To maintain a lowburden study design, we selected only a few statements from each TPB category and elected to include both depression and PTSD in the same statement to minimize assessment length. With regard to attitudes toward intervention, we elected to include the statement "It is important to overcome depressed mood." In the category of social norms, we included several statements, e.g., "I would be embarrassed if my friends knew that I was getting professional help for PTSD or depression.” For selfefficacy, we included several statements, e.g., "I can change my depressed mood by changing my behavior." We utilized the statement "If my doctor told me that I had PTSD or depression, I could accept that" as a statement of "intention to accept diagnosis." We recognize this statement does not by itself predict subsequent treatment but have found it to be highly correlated with past use of mental health services by primary care patients with depression [38]. Table 3 provides the full list of items.

\section{Statistical Analysis}

For descriptive statistics, we reported, as appropriate, either mean \pm standard deviation for continuous variables or frequency and percentage for categorical variables. We report pre and post comparisons with intention-to-treat (all participants enrolled), per-protocol (participants available for analysis), and completer (participants who completed $\geq 4$ modules) analyses. For the intention-to-treat analyses, we input missing data by carrying the last observation forward. We used paired $t$-tests to compare the continuous measures and McNemar test for the categorical measures. We calculated Cohen $d$ (effect size [ES]) for the main study outcomes by comparing baseline and follow-up measures [39]. We performed the analyses with STATA, version 10.0 (StataCorp; College Station, Texas).

\section{RESULTS}

\section{Study Sample}

A total of 708 participants completed the screening CES-D 20, of whom 457 (65\%) met initial eligibility screening (404 did not respond to requests for eligibility assessment). Of the participants, 53 completed telephone eligibility assessment and 50 (11\% of those passing initial eligibility assessment) were eligible and enrolled. The study sample (Table 4) had an average age of almost $30 \mathrm{yr}$ and was diverse with greater than one-quarter ethnic minority representation. Almost 90 percent were male. A majority of participants did not hold four-year college degrees (40 [80\%], not shown in Table 4), slightly more than half were single or divorced, and most had household annual incomes of $\$ 20,000-\$ 39,000$. More than half were either unemployed or students. Almost 88 percent had never received a diagnosis of depression.

\section{Attrition}

The follow-up survey completion percentages were $38(76 \%)$ at week $4,37(74 \%)$ at week 8, $41(82 \%)$ at week 12, and 28 (58\%) for the poststudy attitudes questionnaire. Follow-up clinical measurements of at least one time point were available for 100 percent of participants. Fischer exact and one-way analysis of variance (ANOVA) indicated no significant predictors (such as race, sex, age, education, marital status, or military branch) on attrition.

\section{Feasibility}

Participants demonstrated strong participation in and adherence to the intervention, completing $4.04 \pm 2.45$ e-lessons and $6.02 \pm 5.06$ chats and typing 3,025 $\pm 3,410$ characters in the chats. Of the participants, 33 (66\%) classified as completer, finishing four of six modules. The maximum number of chats for one participant was 17 , with a minimum number of 0 chats. The mean number of characters typed by participants per chat was 392.3. Satisfaction ratings were in the "agree" range for five of the seven items and nearly so for the other two items (Table 2). For example, for the item "I would recommend VETS PREVAIL to a friend dealing with combat or postdeployment stress," participants reported "strong" agreement (4.54 \pm 0.89 ).

\section{Symptoms}

\section{Depressed Mood}

Table 5 shows that depressed mood, as measured by the CES-D 10 in the intention-to-treat analysis, significantly declined from baseline (week 0 ) to week 4 and from baseline to week 12, but not from baseline to week 8. A similar significance pattern occurred for the completer analysis, while the per-protocol analysis showed only a 
Table 3.

Comparisons for attitudes toward mental health services.

\begin{tabular}{|c|c|c|c|c|c|}
\hline \multirow{2}{*}{ Analysis } & \multicolumn{2}{|c|}{ Baseline } & \multicolumn{2}{|c|}{ Week 12} & \multirow{2}{*}{$p$-Value } \\
\hline & $n$ & Mean \pm SD & $n$ & Mean \pm SD & \\
\hline \multicolumn{6}{|l|}{ Intention-to-Treat } \\
\hline \multicolumn{6}{|l|}{ Intention to Accept Diagnosis } \\
\hline It is important to overcome depressed mood. & 50 & $4.52 \pm 0.76$ & 50 & $4.54 \pm 0.79$ & 0.51 \\
\hline $\begin{array}{l}\text { I would be willing to consider face-to-face counseling if I had symptoms of depression or } \\
\text { PTSD. }\end{array}$ & 50 & $4.02 \pm 1.02$ & 50 & $4.02 \pm 1.13$ & 0.80 \\
\hline $\begin{array}{l}\text { I would be embarrassed if my friends knew that I was getting professional help for PTSD } \\
\text { or depression. }\end{array}$ & 50 & $4.18 \pm 0.98$ & 50 & $3.24 \pm 1.35$ & $<0.001$ \\
\hline $\begin{array}{l}\text { I would not want my employer to know that I was getting professional help for PTSD or } \\
\text { Depression. }\end{array}$ & 50 & $3.94 \pm 1.30$ & 50 & $3.42 \pm 1.36$ & $<0.001$ \\
\hline If I had depression or PTSD, others would be disappointed in me. & 50 & $3.40 \pm 1.34$ & 50 & $2.58 \pm 1.23$ & $<0.001$ \\
\hline Depression and PTSD are the result of stressful circumstances in a person's life. & 50 & $2.22 \pm 1.07$ & 50 & $3.34 \pm 1.39$ & $<0.001$ \\
\hline \multicolumn{6}{|l|}{ Per-Protocol } \\
\hline \multicolumn{6}{|l|}{ Intention to Accept Diagnosis } \\
\hline If my doctor told me that I had PTSD or depression, I could accept that. & 50 & $2.78 \pm 1.30$ & 31 & $4.03 \pm 0.87$ & $<0.001$ \\
\hline It is important to overcome depressed mood. & 50 & $4.52 \pm 0.76$ & 31 & $4.52 \pm 0.89$ & 0.57 \\
\hline $\begin{array}{l}\text { I would be willing to consider face-to-face counseling if I had symptoms of depression or } \\
\text { PTSD. }\end{array}$ & 50 & $4.02 \pm 1.02$ & 31 & $4.19 \pm 0.98$ & 0.81 \\
\hline \multicolumn{6}{|l|}{ Stigma/Social Norms } \\
\hline $\begin{array}{l}\text { I would be embarrassed if my friends knew that I was getting professional help for PTSD } \\
\text { or depression. }\end{array}$ & 50 & $4.18 \pm 0.98$ & 31 & $2.58 \pm 1.18$ & $<0.001$ \\
\hline $\begin{array}{l}\text { I would not want my employer to know that I was getting professional help for PTSD or } \\
\text { depression. }\end{array}$ & 50 & $3.94 \pm 1.30$ & 31 & $3.16 \pm 1.29$ & 0.01 \\
\hline \multicolumn{6}{|l|}{ Intention to Accept Diagnosis } \\
\hline If my doctor told me that I had PTSD or depression, I could accept that. & 33 & $2.97 \pm 1.29$ & 27 & $4.04 \pm 0.90$ & $<0.001$ \\
\hline It is important to overcome depressed mood. & 33 & $4.55 \pm 0.79$ & 27 & $4.52 \pm 0.94$ & 0.75 \\
\hline $\begin{array}{l}\text { I would be willing to consider face-to-face counseling if I had symptoms of depression or } \\
\text { PTSD. }\end{array}$ & 33 & $4.06 \pm 1.00$ & 27 & $4.11 \pm 1.01$ & 0.99 \\
\hline \multicolumn{6}{|l|}{ Stigma/Social Norms } \\
\hline $\begin{array}{l}\text { I would be embarrassed if my friends knew that I was getting professional help for PTSD } \\
\text { or depression. }\end{array}$ & 33 & $4.03 \pm 1.10$ & 27 & $2.63 \pm 1.08$ & $<0.001$ \\
\hline $\begin{array}{l}\text { I would not want my employer to know that I was getting professional help for PTSD or } \\
\text { depression. }\end{array}$ & 33 & $3.88 \pm 1.32$ & 27 & $3.26 \pm 1.23$ & 0.02 \\
\hline If I had depression or PTSD, others would be disappointed in me. & 33 & $3.18 \pm 1.40$ & 27 & $2.33 \pm 0.92$ & $<0.001$ \\
\hline Depression and PTSD are the result of stressful circumstances in a person's life. & 33 & $2.48 \pm 1.09$ & 27 & $4.11 \pm 0.89$ & $<0.001$ \\
\hline \multicolumn{6}{|l|}{ Mental Health Self-Efficacy } \\
\hline I can change my depressed mood by changing my behavior. & 33 & $3.85 \pm 0.83$ & 27 & $4.04 \pm 0.81$ & 0.46 \\
\hline I understand how my behavior and my habits can affect my mood. & 33 & $4.15 \pm 0.76$ & 27 & $4.33 \pm 0.92$ & 0.14 \\
\hline I can recognize thoughts and behaviors that tend to trigger depressed moods. & 33 & $3.97 \pm 0.95$ & 27 & $3.52 \pm 1.05$ & 0.28 \\
\hline
\end{tabular}


Table 4.

Demographics $(n=49)$.

\begin{tabular}{|c|c|c|}
\hline Characteristic & $n(\%)$ & Mean \pm SD \\
\hline \multicolumn{3}{|l|}{$\overline{\text { Sex }}$} \\
\hline Female & $5(10.20)$ & - \\
\hline Male & $44(89.80)$ & - \\
\hline \multicolumn{3}{|l|}{ Race/Ethnicity } \\
\hline African American & $3(6.12)$ & - \\
\hline Asian & $4(8.16)$ & - \\
\hline Hispanic & $5(10.20)$ & - \\
\hline White & 36 (73.47) & - \\
\hline Other & $1(2.04)$ & - \\
\hline Age (yr) & - & $29.57 \pm 7.17$ \\
\hline Education (yr) & - & $13.31 \pm 2.79$ \\
\hline \multicolumn{3}{|l|}{ Annual Household Income (\$) } \\
\hline$<10,000$ & $1(2.04)$ & - \\
\hline 10,000-19.999 & 7 (14.29) & - \\
\hline 20,000-29,999 & $11(22.45)$ & - \\
\hline $30,000-39,999$ & $14(28.57)$ & - \\
\hline $40,000-49,999$ & $6(12.24)$ & - \\
\hline $50,000-59,999$ & $4(8.16)$ & - \\
\hline $60,000-69,999$ & $2(4.08)$ & - \\
\hline $70,000-79,999$ & $0(0)$ & - \\
\hline $80,000-99,999$ & $0(0)$ & - \\
\hline$>100,000$ & $4(8.16)$ & - \\
\hline \multicolumn{3}{|l|}{ Branch of Service } \\
\hline Air Force & $3(6.12)$ & - \\
\hline Army & $26(53.06)$ & - \\
\hline Marine Corps & $12(24.49)$ & - \\
\hline Navy & $8(16.33)$ & - \\
\hline \multicolumn{3}{|l|}{ Marital Status } \\
\hline Married & $22(44.90)$ & - \\
\hline Single & $20(40.82)$ & - \\
\hline Divorced & 7 (14.29) & - \\
\hline Children (n) & - & $0.90 \pm 1.37$ \\
\hline \multicolumn{3}{|l|}{ Employment } \\
\hline Full-time (not student) & $18(36.73)$ & - \\
\hline Part-time (not student) & $3(6.12)$ & - \\
\hline Unemployed (not student) & $13(26.53)$ & - \\
\hline Student (not working) & $10(20.41)$ & - \\
\hline Student (working) & $5(10.20)$ & - \\
\hline \multicolumn{3}{|l|}{ Family Depression History } \\
\hline Do Not Know & $13(26.53)$ & - \\
\hline Yes & 24 (48.98) & - \\
\hline No & $12(24.49)$ & - \\
\hline \multicolumn{3}{|l|}{ Depression History } \\
\hline Yes & $6(12.24)$ & - \\
\hline No & $43(87.76)$ & - \\
\hline \multicolumn{3}{|l|}{ Depression Treatment } \\
\hline Counseling and Medication & $2(4.08)$ & - \\
\hline Medication Only & $4(8.16)$ & - \\
\hline Not Applicable (no history of depression) & $42(85.71)$ & - \\
\hline No Treatment & $1(2.04)$ & - \\
\hline \multicolumn{3}{|c|}{$\begin{array}{l}\text { Note: Shown for only } 49 \text { participants because } 1 \text { did not complete demograph- } \\
\text { ics information survey. One participant who answered "no" to depression his- } \\
\text { tory item mistakenly responded "no treatment" to depression treatment item } \\
\text { instead of "not applicable." } \\
\text { SD = standard deviation. }\end{array}$} \\
\hline
\end{tabular}

significant decline from baseline to week 4. Regarding the dichotomous outcomes, the percentage of participants with CES-D 10 scores $>9$ in the intention-to-treat analysis did not significantly decline from baseline to weeks 4, 8, or 12 . However, in the per-protocol and completer analyses, the percentage significantly declined from baseline to weeks 4 and 12. The CES-D 10 scores declined from baseline to weeks 4 and 12 with ESs in the moderate range and were generally larger (for baseline to week 12) moving from intention-to-treat $(\mathrm{ES}=-0.32$, 95\% confidence interval $[\mathrm{CI}]=-0.71$ to 0.08$)$ to per-protocol $(\mathrm{ES}=-0.41,95 \% \mathrm{CI}=$ -0.84 to 0.02 ) to completer ( $\mathrm{ES}=-0.59,95 \% \mathrm{CI}=-1.08$ to -0.08) analyses (Table 6). Finally, both CES-D 10 scores and the percentage with clinically significant depressed mood significantly declined from screening to baseline $(p<$ 0.001 , Table 5). Fisher exact and one-way ANOVA indicated no significant predictors (such as race, sex, age, education, marital status, or military branch) of CES-D 10 score outcomes for any of the time periods (weeks 4,8 , and 12) postintervention.

\section{Posttraumatic Stress}

Table 5 shows that symptoms of posttraumatic stress in the intention-to-treat analysis significantly declined from baseline (week 0) to weeks 4, 8, and 12. A similar significance pattern occurred in the per-protocol and completer analyses. Regarding the dichotomous outcomes, the percentage of participants with PCL-M scores $>30$ in the intention-to-treat analysis significantly declined from baseline to week 12. We did not see this significance pattern in the perprotocol and completer analyses. PCL-M scores declined from baseline to weeks 4, 8, and 12 with ESs in the moderate range and were generally larger with greater time (baseline to week 12) and moving from intention-to-treat (ES $=-0.30,95 \% \mathrm{CI}=-0.69$ to 0.10 ) to per-protocol (ES $=-0.53,95 \% \mathrm{CI}=-0.77$ to 0.08 ) to completer (ES = $-0.57,95 \%$ CI $=-1.06$ to -0.06 ) analyses (Table 6). Fisher exact and one-way ANOVA indicated no significant predictors (such as race, sex, age, education, marital status, or military branch) of PCL-M outcomes for any of the time periods (weeks 4,8 , and 12) postintervention.

\section{Functional Status}

Table 5 shows that no significant changes occurred for functional status for any of the analyses. ESs were small for the SF-12 mental component score but were gradually larger with time (baseline to week 12) and moving from 
JRRD, Volume 49, Number 8, 2012

Table 5.

Mental health symptom and functional status outcomes.

\begin{tabular}{|c|c|c|c|c|c|c|c|c|c|c|c|c|c|c|c|c|}
\hline \multirow{2}{*}{ Analysis Outcomes } & \multicolumn{2}{|r|}{ Screening } & \multicolumn{2}{|r|}{ Week 0} & \multicolumn{2}{|r|}{ Week 4} & \multicolumn{2}{|r|}{ Week 8} & \multicolumn{2}{|r|}{ Week 12} & \multicolumn{2}{|c|}{ Week 0-4 } & \multicolumn{2}{|c|}{ Week 0-8 } & \multicolumn{2}{|c|}{ Week 0-12 } \\
\hline & $n$ & Mean \pm SD & $n$ & Mean \pm SD & $n$ & Mean \pm SD & $n$ & Mean \pm SD & $n$ & Mean \pm SD & $n$ & $\begin{array}{c}p \text { - } \\
\text { Value }\end{array}$ & $n$ & $\begin{array}{c}p \text { - } \\
\text { Value }\end{array}$ & $n$ & $\begin{array}{c}p- \\
\text { Value }\end{array}$ \\
\hline \multicolumn{17}{|l|}{ Intention-to-Treat } \\
\hline CES-D 10 (score) & 50 & $11.8 \pm 3.5$ & 50 & $8.9 \pm 4.0$ & 50 & $7.3 \pm 4.2$ & 50 & $7.8 \pm 4.4$ & 50 & $7.4 \pm 5.3$ & 50 & 0.01 & 50 & 0.33 & 50 & 0.04 \\
\hline CES-D 10 (score >9) & 50 & $(74.0 \pm 37.0)$ & 50 & $(40.0 \pm 20.0)$ & 50 & $(38.0 \pm 19.0)$ & 50 & $(50.0 \pm 25.0)$ & 50 & $(32.0 \pm 16.0)$ & 50 & 1.00 & 50 & 0.36 & 50 & 0.50 \\
\hline PCL-M (score > 30) & 50 & 一 & 50 & $(64.0 \pm 32.0)$ & 50 & $(56.0 \pm 28.0)$ & 50 & $(52.0 \pm 26.0)$ & 50 & $(44.0 \pm 22.0)$ & 50 & 0.39 & 50 & 0.18 & 50 & 0.03 \\
\hline \multicolumn{17}{|l|}{ Functional Status } \\
\hline SF-12 Mental (score) & 50 & 一 & 50 & $44.6 \pm 10.8$ & 50 & $44.5 \pm 11.4$ & 50 & $45.3 \pm 10.6$ & 50 & $46.3 \pm 11.6$ & 50 & 0.85 & 50 & 0.37 & 50 & 0.10 \\
\hline SF-12 Physical (score) & 50 & - & 50 & $52.5 \pm 6.8$ & 50 & $52.9 \pm 5.9$ & 50 & $53.0 \pm 6.2$ & 50 & $52.2 \pm 6.5$ & 50 & 0.81 & 50 & 0.94 & 50 & 0.20 \\
\hline
\end{tabular}

\section{Per-Protocol}

Depressive Disorder

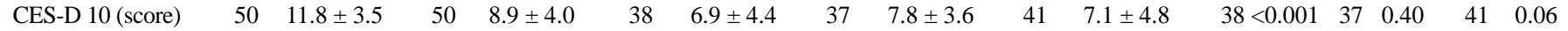

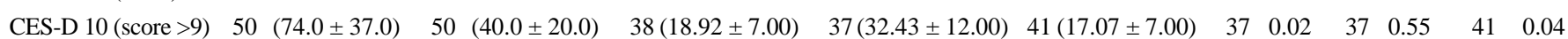
PTSD

$\begin{array}{lllllllllllllllll}\text { PCL-M (score) } & 50 & - & 50 & 35.0 \pm 10.8 & 38 & 30.0 \pm 9.7 & 37 & 31.7 \pm 10.8 & 41 & 31.1 \pm 11.6 & 39 & 0.01 & 37 & 0.04 & 41 & 0.05 \\ \text { PCL-M (score }>30) & 50 & - & 50 & (64.0 \pm 32.0) & 39(53.85 \pm 21.00) & 37(51.35 \pm 19.00) & 41(41.46 \pm 17.00) & 39 & 0.39 & 37 & 0.23 & 41 & 0.08 \\ \text { Functional Status } & & & & & & & & & & & & & & & & \\ \text { SF-12 Mental (score) } & 50 & - & 50 & 44.6 \pm 10.8 & 39 & 45.8 \pm 9.8 & 36 & 46.3 \pm 9.0 & 41 & 47.1 \pm 10.5 & 39 & 0.91 & 36 & 0.29 & 41 & 0.09 \\ \text { SF-12 Physical (score) } & 50 & - & 50 & 52.5 \pm 6.8 & 39 & 52.7 \pm 5.7 & 36 & 52.5 \pm 6.4 & 41 & 52.0 \pm 6.7 & 39 & 0.73 & 36 & 0.74 & 41 & 0.16\end{array}$

\section{Completer}

Depressive Disorder

$\begin{array}{lllllllllllllll}\text { CES-D } 10 \text { (score) } \quad 33 & 11.5 \pm 3.0 & 33 & 9.3 \pm 4.2 & 33 & 6.7 \pm 3.7 & 30 & 7.5 \pm 3.5 & 31 & 6.8 \pm 4.3 & 33<0.001 & 30 & 0.11 & 31 & 0.02\end{array}$

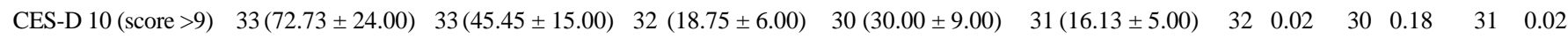
PTSD

$\begin{array}{lllllllllllllllll}\text { PCL-M (score) } & 33 & - & 33 & 33.6 \pm 9.7 & 33 & 30.2 \pm 7.8 & 30 & 29.5 \pm 7.3 & 31 & 28.5 \pm 8.1 & 33 & 0.02 & 30 & 0.02 & 31 & 0.01 \\ \text { PCL-M (score > 30) } & 33 & - & 33(60.61 \pm 20.00) & 33(51.52 \pm 17.00) & 30(46.67 \pm 14.00) & 31(35.48 \pm 11.00) & 33 & 0.51 & 30 & 0.34 & 31 & 0.09 \\ \text { Functional Status } & & & & & & & & & & & & & & & & \\ \text { SF-12 Mental (score) } & 33 & - & 33 & 45.5 \pm 8.9 & 33 & 46.6 \pm 9.2 & 30 & 47.6 \pm 6.8 & 31 & 48.1 \pm 9.2 & 33 & 0.32 & 30 & 0.12 & 31 & 0.13 \\ \text { SF-12 Physical (score) } & 33 & - & 33 & 51.7 \pm 7.2 & 33 & 57.2 \pm 5.9 & 30 & 53.2 \pm 6.1 & 31 & 53.0 \pm 5.9 & 33 & 0.92 & 30 & 0.64 & 31 & 0.96\end{array}$

Note: $p$-Values based on 95\% confidence interval. $p$-Value comparison from screening to week 0 for CES-D 10 outcomes: $<0.001$.

CES-D = Center for Epidemiologic Studies-Depression, PCL-M = PTSD Checklist-Military version, PTSD = posttraumatic stress disorder, SD = standard deviation, SF-12 = 12-Item Short Form Health Survey.

intention-to-treat $(\mathrm{ES}=0.15,95 \% \mathrm{CI}=-0.24$ to 0.54$)$ to per-protocol ( $\mathrm{ES}=0.23,95 \% \mathrm{CI}=-0.19$ to 0.65$)$ to completer ( $\mathrm{ES}=0.29,95 \% \mathrm{CI}=-0.21$ to 0.54 ) analyses (Table 6).

\section{Attitudes Toward Treatment Seeking}

Improvement occurred for the areas of intention to accept treatment and perceived social norms/stigma but not for mental health self-efficacy in managing mental disorder symptoms. Similar significance patterns occurred for all three analytical approaches. With regard to intention to accept treatment, participants indicated much stronger agreement at week 12 than at baseline with the statement "If my doctor told me that I had PTSD or depression, I could accept that” ( $p<0.001$, Table 3 ). However, we saw no change in willingness to accept faceto-face counseling. With regard to perceived social norms/stigma, we noted significant change for all items assessed. Participants were more willing to accept a diagnosis after being exposed to the program, with ESs ranging from moderate in intention-to-treat $(\mathrm{ES}=0.47$, $95 \% \mathrm{CI}=0.07$ to 0.87 ) to larger in per-protocol (ES = $1.08,95 \% \mathrm{CI}=0.59$ to 1.55$)$ and completer ( $\mathrm{ES}=0.95$, $95 \%$ CI $=0.40$ to 1.47 ) analyses. The ESs were also large 
Table 6.

Pre/post effect sizes (ESs) for main outcomes.

\begin{tabular}{|c|c|c|c|c|c|c|}
\hline \multirow{2}{*}{ Measure } & \multicolumn{2}{|c|}{ Week 0-4 } & \multicolumn{2}{|c|}{ Week 0-8 } & \multicolumn{2}{|c|}{ Week 0-12 } \\
\hline & ES & CI & ES & CI & ES & CI \\
\hline \multicolumn{7}{|l|}{ Intention-to-Treat Analysis } \\
\hline PCL-M & -0.27 & -0.66 to 0.13 & -0.28 & -0.67 to 0.12 & -0.30 & -0.69 to 0.10 \\
\hline SF-12 (Mental) & -0.01 & -0.40 to 0.38 & 0.07 & -0.33 to 0.46 & 0.15 & -0.24 to 0.54 \\
\hline \multicolumn{7}{|l|}{ Per-Protocol Analysis } \\
\hline CES-D 10 & -0.48 & -0.91 to -0.04 & -0.29 & -0.71 to 0.14 & -0.41 & -0.84 to 0.02 \\
\hline PCL-M & -0.48 & -0.91 to -0.05 & -0.31 & -0.73 to 0.12 & -0.53 & -0.77 to 0.08 \\
\hline SF-12 (Mental) & 0.12 & -0.31 to 0.54 & 0.17 & -0.26 to 0.60 & 0.23 & -0.19 to 0.65 \\
\hline CES-D 10 & -0.66 & -1.14 to -0.15 & -0.46 & -0.96 to 0.04 & -0.59 & -1.08 to -0.08 \\
\hline PCL-M & -0.39 & -0.87 to 0.11 & -0.47 & -0.97 to 0.03 & -0.57 & -1.06 to -0.06 \\
\hline SF-12 (Mental) & 0.12 & -0.36 to 0.60 & 0.26 & -0.24 to 0.76 & 0.29 & -0.21 to 0.78 \\
\hline Willingness to accept & - & - & - & - & 0.95 & 0.40 to 1.47 \\
\hline Embarrassed if my friends knew & - & - & - & - & -1.28 & -1.82 to -0.71 \\
\hline
\end{tabular}

CES-D = Center for Epidemiologic Studies-Depression, CI = confidence interval, PCL-M = Posttraumatic Stress Disorder Checklist-Military version, SF-12 = 12-Item Short Form Health Survey.

for changes related to stigma (decreased agreement with statement "I would be embarrassed if my friends knew that I was getting professional help for PTSD or depression”) in the intention-to-treat analysis (ES $=0.80,95 \%$ $\mathrm{CI}=1.20$ to 0.38 ) and even larger in the per-protocol $(\mathrm{ES}=1.51,95 \% \mathrm{CI}=0.99$ to 2.00$)$ and completer (ES = $1.28,95 \% \mathrm{CI}=0.71,1.87)$ analyses.

\section{Adverse Events/Evidence of Harm}

We detected only one incident of self-harm ideation during the study, and this occurred before enrollment. No other events were reported. Also, we provided servicemembers who reported depressed mood above a CES-D 10 score of 16 or who experienced depressed mood increases with recommendations to seek face-to-face evaluations at their local medical center.

\section{DISCUSSION}

A diverse group of veterans and servicemembers, nearly all of whom had never been diagnosed with or treated for depression, adhered to and reported generally favorable ratings of satisfaction with the VETS PREVAIL intervention. Two-thirds of the participants completed the intervention, and participants rated in the agree range for most items in the domain of sociocultural relevance, supporting hypothesis 1 . Consistent with hypothesis 2, improvements in depression and PTSD symptoms were in the moderate ES range and were sustained to week 12 postenrollment. Contrary to hypothesis 3, there were no significant improvements in functional status. Finally, participants reported much more positive attitudes regarding willingness to accept a mental health diagnosis and perceived social norms/stigma, providing strong support for hypothesis 4.

To our knowledge, this is the first report of a study using a transdiagnostic approach to evaluate the feasibility of an Internet-based intervention developed specifically for combat veterans. Participation and sociocultural relevance and satisfaction ratings for this intervention are comparable or superior to those observed for other Internet interventions. VETS PREVAIL participants would "recommend the intervention to a friend" (5-point Likert scale, where 1 = strongly disagree and 5 = strongly agree) with mean scores of 4.52 versus 4.32 for CATCH-IT participants 
(online plus face-to-face adolescent depression prevention) [40]. Dropout rates range from 50 to 100 percent on Internet interventions compared with 100 percent retention in this study for at least one follow-up [41-42]. The percentage of participants visiting the Web site at least once was comparable to an intervention that has considerable face-toface engagement with a primary care physician $(82 \%$ VETS PREVAIL vs 85\% CATCH-IT) [40]. There are also similarities in number of characters typed (VETS PREVAIL mean: 3,025 vs CATCH-IT mean: 3,580) [40]. These favorable adherence and ratings of sociocultural relevance could be explained by (1) extensive sociocultural adaptation and individual tailoring [27] or (2) selection of favorably disposed participants. Conjecture 1 is supported by the large favorable changes in stigma regarding mental health treatment and willingness to accept diagnosis observed in this study. This substantial shift toward more favorable views of mental health treatment suggests the intervention itself was very well received. However, the relatively low recruitment rate of 7 percent (vs $28 \%-40 \%$ in face-to-face recruitment models) suggests a selection bias may have played a role, supporting conjecture 2 [43-44]. However, these low recruitment rates may not be unusual for a fully online intervention and may be consistent with the finding that only 10 to 20 percent of adults are interested in an Internet-based lifestyle change at any given time [45-46].

Furthermore, the moderate depression pre/post ESs were less than those seen in treatment interventions involving therapist and discussion group contact (VETS PREVAIL ES $=0.45$ vs Internet plus therapist contact ES = 0.93) [21,47-48] but comparable with preventive interventions using an Internet-only approach (male adolescents $\mathrm{ES}=0.29)[47,49]$. Similarly, this intervention demonstrated pre/post ESs comparable to an Internet-only PTSD intervention but lower than those reported in one with therapist contact (VETS PREVAIL ES $=0.30$ vs ES $=0.45$ for Internet-only or ES $=0.76$ for Internet plus therapist) [50-51]. The ESs presented here may have also been affected by the lower education and income levels of the study population, who were encountering significant adjustment challenges. However, the increasing ESs from intention-to-treat to per-protocol and finally to completer analyses suggest that those who were more fully engaged with the intervention experienced greater gains [52]. The significant change in CES-D 10 scores from screening to baseline suggests the possibility that positive expectancies or nonspecific intervention effects may explain some pre/ post changes. Conversely, these initial improvements before the baseline assessment, which in fact may relate to contact with the intervention Web site itself and/or its associated Facebook page, may have reduced observed pre/post changes during the formal study observation period [53].

This is the first report of which we are aware of an Internet-based intervention demonstrating favorable changes in participants' social norms or intention to accept a mental disorder diagnosis. The ES reported that improvement in care seeking, social norms, and/or intentions to accept diagnosis for mental disorders is much larger than that observed in other Internet-based interventions (VETS PREVAIL ES $=0.98$ for intention to accept diagnosis and $\mathrm{ES}=0.80$ for stigma related to friends vs $\mathrm{ES}=0.11-0.12$ for standard CBT interventions for mental health care seeking attitudes) [54]. In this sample, attitudes at baseline before treatment were more "negative" than community samples, possibly reflecting male and military values of stoicism, which are thought to be major barriers for obtaining mental health services $[3,55]$. Perhaps the opportunity to "try out" approaches within an ostensibly military cultural framework was particularly influential, especially when accompanied by peer support [56]. This conjecture is based on our previously reported findings that attitudes toward depression care seeking are strongly influenced by perceived helpfulness of past care episodes as well as the opinions of peers $[13,36]$.

\section{LIMITATIONS}

It is important to recognize several limitations to the aforementioned findings. First, we do not know whether some of the study participants may have sought treatment during the study, and if so, whether some of the pre/post changes resulted from such treatment. Similarly, we did not have a control group, so we do not know to what extent the changes observed reflect natural history. However, the period of waiting between screening and study enrollment, during which CES-D 10 scores declined, may have acted as a "wash out" period that separated expectancy and attention effects from those associated with the formal intervention.

With respect to the items related to attitudes toward treatment seeking, the main limitations were (1) use of double-barreled statements (depression and PTSD) makes it impossible to evaluate responses in the context of a particular disorder; (2) the number of statements used in most categories was restricted, with the goal of minimizing study burden; and (3) the statement used for intention to 
accept diagnosis could have been more carefully written to assess intention for treatment seeking.

Other important limitations include the small sample size, short-term follow-up, and lack of poststudy interviews to provide a more comprehensive picture of the participant experience. The online recruitment can be considered both a limitation in study design, allowing only a limited knowledge of the circumstances and settings from which participants were recruited, as well as a strength, considering ease of implementation. It is unclear what the total net effect of the online recruiting methods was. For example, engagement in primary care or mental health specialty settings could offer the prospect of recruiting less motivated persons but the supportive care context might also spur participation [57].

\section{CONCLUSIONS}

These results suggest that Internet interventions adapted from standard psychotherapy practices can be constructed so as to engage at least some portion of the returning servicemember population that is experiencing symptoms of depression and PTSD. Internet-based models could offer both indicated prevention and early treatment outside the formal system of care or within either primary care or mental health clinic intake programs. The intervention may benefit from the addition of cognitive elements, including thought change for depression and narrative revision for PTSD, given that the current intervention was well received and found to be culturally acceptable. Such additions hold the prospect of further increasing ESs and perhaps finding functional status changes. Follow-up studies could include demonstration projects evaluating the effect of VETS PREVAIL in health promotion within defined populations and/or randomized clinical trials comparing the intervention with wait-list or usual care conditions to determine the degree to which the intervention benefits postdeployment servicemembers and veterans over and above these "naturalistic" conditions. An important next step will be determining how to increase the proportion of those initially screened who formally enroll in the intervention, a key challenge for all Internet-based interventions.

\section{ACKNOWLEDGMENTS}

Author Contributions:

Study design: B. W. Van Voorhees.
Study supervision: B. W. Van Voorhees.

Development of core BA components in intervention: J. Gollan.

Supervising statistician: J. Fogel.

Financial Disclosures: Dr. Van Voorhees, as President of RISE Consulting, has served as a consultant to Prevail Health Solutions (Chicago, Illinois), Mevident Inc (San Francisco, California), Social Kinetics (Palo Alto, California), and the University of Hong Kong (Hong Kong) to develop Internet-based interventions. RISE Consulting has received a total of $\$ 43,262.50$ from Prevail Health Solutions (2008-2010) for providing advice in Internet program design and interventions for mental disorders, grant writing and editing, and supervision and analysis of the phase 1 clinical trial presented in this article. The total compensation received since initiation of this study is $\$ 23,500.00$. Prevail Health Solutions was formed while the founders (Richard Gengler, MBA, and Roger Sweis, MBA) were attending the University of Chicago Booth School of Business (Chicago, Illinois). The founders developed an initial business plan for course work that later grew into Prevail Health Solutions. During this time, Dr. Van Voorhees served as academic scientific advisor with regard to Internetbased mental health programs. After the founders completed their graduate studies, Dr. Van Voorhees continued to work (as a paid consultant) with Prevail Health Solutions as an outside scientific advisor. Dr. Van Voorhees is an Assistant Professor of Medicine, Pediatrics and Psychiatry at the University of Chicago, but the University was not a party to this study. Dr. Gollan was paid as a consultant $(\$ 3,792.50)$ between November 2008 and June 2009 by Prevail Health Solutions to help develop the intervention. Dr. Fogel was paid as a consultant $(\$ 2,875.00)$ between September 2008 and August 2010 by Prevail Health Solutions as the statistical consultant.

Funding/Support: This material was based on work supported by a grant from the McCormick Foundation.

Institutional Review: This study was approved by the Quorum Institutional Review Board (24633-1).

Participant Follow-Up: Participants will be notified of the publication of this study via the email address provided during study enrollment.

\section{REFERENCES}

1. Iversen AC, van Staden L, Hughes JH, Browne T, Hull L, Hall J, Greenberg N, Rona RJ, Hotopf M, Wessely S, Fear NT. The prevalence of common mental disorders and PTSD in the UK military: using data from a clinical interview-based study. BMC Psychiatry. 2009;9:68. [PMID:19878538]

http://dx.doi.org/10.1186/1471-244X-9-68

2. Seal KH, Metzler TJ, Gima KS, Bertenthal D, Maguen S, Marmar CR. Trends and risk factors for mental health diagnoses among Iraq and Afghanistan veterans using Department of Veterans Affairs health care, 2002-2008. Am J Public Health. 2009;99(9):1651-58. [PMID:19608954] http://dx.doi.org/10.2105/AJPH.2008.150284

3. Hoge CW, Castro CA, Messer SC, McGurk D, Cotting DI, Koffman RL. Combat duty in Iraq and Afghanistan, mental health problems, and barriers to care. N Engl J Med. 2004; 
351(1):13-22. [PMID:15229303]

http://dx.doi.org/10.1056/NEJMoa040603

4. Van Staden L, Fear NT, Iversen AC, French CE, Dandeker C, Wessely S. Transition back into civilian life: a study of personnel leaving the U.K. armed forces via "military prison”. Mil Med. 2007;172(9):925-30. [PMID:17937354]

5. Hilton MF, Scuffham PA, Vecchio N, Whiteford HA. Using the interaction of mental health symptoms and treatment status to estimate lost employee productivity. Aust N Z J Psychiatry. 2010;44(2):151-61. [PMID:20113304]

http://dx.doi.org/10.3109/00048670903393605

6. Feldner MT, Monson CM, Friedman MJ. A critical analysis of approaches to targeted PTSD prevention: current status and theoretically derived future directions. Behav Modif. 2007;31(1):80-116. [PMID:17179532]

http://dx.doi.org/10.1177/0145445506295057

7. Garber J. Depression in children and adolescents: linking risk research and prevention. Am J Prev Med. 2006;31(6 Suppl 1):S104-25. [PMID:17175406] http://dx.doi.org/10.1016/j.amepre.2006.07.007

8. Phillips CJ, Leardmann CA, Gumbs GR, Smith B. Risk factors for posttraumatic stress disorder among deployed US male Marines. BMC Psychiatry. 2010;10:52. [PMID:20579379] http://dx.doi.org/10.1186/1471-244X-10-52

9. LeardMann CA, Smith TC, Smith B, Wells TS, Ryan MA; Millennium Cohort Study Team. Baseline self reported functional health and vulnerability to post-traumatic stress disorder after combat deployment: prospective US military cohort study. BMJ. 2009;338:b1273. [PMID:19372117] http://dx.doi.org/10.1136/bmj.b1273

10. Humensky J, Kuwabara SA, Fogel J, Wells C, Goodwin B, Van Voorhees BW. Adolescents with depressive symptoms and their challenges with learning in school. J Sch Nurs. 2010;26(5):377-92. [PMID:20606058] http://dx.doi.org/10.1177/1059840510376515

11. Lewinsohn PM, Gotlib IH, Seeley JR. Depression-related psychosocial variables: are they specific to depression in adolescents? J Abnorm Psychol. 1997;106(3):365-75.

[PMID:9241938]

http://dx.doi.org/10.1037/0021-843X.106.3.365

12. Van Voorhees BW, Walters AE, Prochaska M, Quinn MT. Reducing health disparities in depressive disorders outcomes between non-Hispanic Whites and ethnic minorities: a call for pragmatic strategies over the life course. Med Care Res Rev. 2007;64(5 Suppl):157S-94S. [PMID:17766647] http://dx.doi.org/10.1177/1077558707305424

13. Van Voorhees BW, Fogel J, Houston TK, Cooper LA, Wang NY, Ford DE. Attitudes and illness factors associated with low perceived need for depression treatment among young adults. Soc Psychiatry Psychiatr Epidemiol. 2006;41(9):
746-54. [PMID:16896516]

http://dx.doi.org/10.1007/s00127-006-0091-X

14. Landback J, Prochaska M, Ellis J, Dmochowska K, Kuwabara SA, Gladstone T, Larson J, Stuart S, Gollan J, Bell C, Bradford N, Reinecke M, Fogel J, Van Voorhees BW. From prototype to product: development of a primary care/ internet based depression prevention intervention for adolescents (CATCH-IT). Community Ment Health J. 2009;45(5): 349-54.

[PMID:19641992]

http://dx.doi.org/10.1007/s10597-009-9226-3

15. Ritterband LM, Thorndike FP, Cox DJ, Kovatchev BP, Gonder-Frederick LA. A behavior change model for internet interventions. Ann Behav Med. 2009;38(1):18-27.

[PMID:19802647]

http://dx.doi.org/10.1007/s12160-009-9133-4

16. Andersson G, Cuijpers P. Internet-based and other computerized psychological treatments for adult depression: a metaanalysis. Cogn Behav Ther. 2009;38(4):196-205.

[PMID:20183695]

http://dx.doi.org/10.1080/16506070903318960

17. Benight CC, Ruzek JI, Waldrep E. Internet interventions for traumatic stress: a review and theoretically based example. J Trauma Stress. 2008;21(6):513-20. [PMID:19107724] http://dx.doi.org/10.1002/jts.20371

18. Van Voorhees BW, Mahoney N, Mazo R, Barrera AZ, Siemer CP, Gladstone TR, Munoz RF. Internet-based depression over the life course: a call for behavioral vaccines. Pyschiatr Clin North Am. 2011;34(1):167-83.

[PMID:21333846] http://dx.doi.org/10.1016/j.psc.2010.11.002

19. Gortner ET, Gollan JK, Dobson KS, Jacobson NS. Cognitive-behavioral treatment for depression: relapse prevention. J Consult Clin Psychol. 1998;66(2):377-84.

[PMID:9583341] http://dx.doi.org/10.1037/0022-006X.66.2.377

20. Cuijpers P, van Straten A, Warmerdam L. Problem solving therapies for depression: a meta-analysis. Eur Psychiatry. 2007;22(1):9-15. [PMID:17194572] http://dx.doi.org/10.1016/j.eurpsy.2006.11.001

21. Cuijpers P, Donker T, van Straten A, Li J, Andersson G. Is guided self-help as effective as face-to-face psychotherapy for depression and anxiety disorders? A systematic review and meta-analysis of comparative outcome studies. Psychol Med. 2010;40(12):1943-57. [PMID:20406528] http://dx.doi.org/10.1017/S0033291710000772

22. Griffiths KM, Calear AL, Banfield M. Systematic review on Internet Support Groups (ISGs) and depression (1): Do ISGs reduce depressive symptoms? J Med Internet Res. 2009;11(3):e40. [PMID:19793719] http://dx.doi.org/10.2196/jmir.1270 
VAN VOORHEES et al. Online intervention for mental distress

23. Griffiths KM, Calear AL, Banfield M, Tam A. Systematic review on Internet Support Groups (ISGs) and depression (2): What is known about depression ISGs? J Med Internet Res. 2009;11(3):e41. [PMID:19793718] http://dx.doi.org/10.2196/jmir.1303

24. Miller WR, Rollnick S. Motivational interviewing: Preparing people to change addictive behavior. New York (NY): Guilford Press; 1991.

25. Vansteenkiste M, Sheldon KM. There's nothing more practical than a good theory: integrating motivational interviewing and self-determination theory. Br J Clin Psychol. 2006;45(Pt 1):63-82. [PMID:16480567] http://dx.doi.org/10.1348/014466505X34192

26. Van Voorhees BW, Ellis JM, Gollan JK, Bell CC, Stuart SS, Fogel J, Corrigan PW, Ford DE. Development and process evaluation of a primary care internet-based intervention to prevent depression in emerging adults. Prim Care Companion J Clin Psychiatry. 2007;9(5):346-55. [PMID:17998953] http://dx.doi.org/10.4088/PCC.v09n0503

27. Resnicow K, Davis RE, Zhang G, Konkel J, Strecher VJ, Shaikh AR, Tolsma D, Calvi J, Alexander G, Anderson JP, Wiese C. Tailoring a fruit and vegetable intervention on novel motivational constructs: results of a randomized study. Ann Behav Med. 2008;35(2):159-69. [PMID:18401673] http://dx.doi.org/10.1007/s12160-008-9028-9

28. Hoge CW, Castro CA, Messer SC, McGurk D, Cotting DJ, Koffman RJ. Combat duty in Iraq and Afghanistan, mental health problems and barriers to care. US Army Med Dep J. 2008;7-17.

29. John R, Buschman P, Chaszar M, Honig J, Mendonca E, Bakken S. Development and evaluation of a PDA-based decision support system for pediatric depression screening. Stud Health Technol Inform. 2007;129(Pt 2):1382-86. [PMID:17911940]

30. Bliese PD, Wright KM, Adler AB, Cabrera O, Castro CA, Hoge CW. Validating the primary care posttraumatic stress disorder screen and the posttraumatic stress disorder checklist with soldiers returning from combat. J Consult Clin Psychol. 2008;76(2):272-81. [PMID:18377123] http://dx.doi.org/10.1037/0022-006X.76.2.272

31. Radloff LS. The CES-D Scale: a self-report depression scale for research in the general population. Appl Psychol Meas. 1977;1(3):385-401.

http://dx.doi.org/10.1177/014662167700100306

32. Andresen EM, Malmgren JA, Carter WB, Patrick DL. Screening for depression in well older adults: evaluation of a short form of the CES-D (Center for Epidemiologic Studies Depression Scale). Am J Prev Med. 1994;10(2):77-84. [PMID:8037935]

33. McHorney CA, Ware JE Jr, Raczek AE. The MOS 36-Item Short-Form Health Survey (SF-36): II. Psychometric and clinical tests of validity in measuring physical and mental health constructs. Med Care. 1993;31(3):247-63.

[PMID:8450681]

http://dx.doi.org/10.1097/00005650-199303000-00006

34. Cooper LA, Gonzales JJ, Gallo JJ, Rost KM, Meredith LS, Rubenstein LV, Wang NY, Ford DE. The acceptability of treatment for depression among African-American, Hispanic, and white primary care patients. Med Care. 2003; 41(4):479-89. [PMID:12665712] http://dx.doi.org/10.1097/01.MLR.0000053228.58042.E4

35. Cooper-Patrick L, Powe NR, Jenckes MW, Gonzales JJ, Levine DM, Ford DE. Identification of patient attitudes and preferences regarding treatment of depression. J Gen Intern Med. 1997;12(7):431-38. [PMID:9229282] http://dx.doi.org/10.1046/j.1525-1497.1997.00075.x

36. Van Voorhees BW, Fogel J, Houston TK, Cooper LA, Wang NY, Ford DE. Beliefs and attitudes associated with the intention to not accept the diagnosis of depression among young adults. Ann Fam Med. 2005;3(1):38-46. [PMID:15671189] http://dx.doi.org/10.1370/afm.273

37. Ajzen I. The directive influence of attitudes on behavior. In: Gollwitzer PM, Bargh JA, editors. The psychology of action: Linking cognition and motivation to behavior. New York (NY): Guilford Press; 1996. p. 385-403.

38. Van Voorhees BW, Cooper LA, Rost KM, Nutting P, Rubenstein LV, Meredith L, Wang NY, Ford DE. Primary care patients with depression are less accepting of treatment than those seen by mental health specialists. J Gen Intern Med. 2003;18(12):991-1000. [PMID:14687257] http://dx.doi.org/10.1111/j.1525-1497.2003.21060.x

39. Cohen J. Statistical power analysis for the behavioral sciences. 2nd ed. Hillsdale (NJ): L. Erlbaum Associates; 1988.

40. Van Voorhees BW, Fogel J, Pomper BE, Marko M, Reid N, Watson N, Larson J, Bradford N, Fagan B, Zuckerman S, Wiedmann P, Domanico R. Adolescent dose and ratings of an Internet-based depression prevention program: A randomized trial of primary care physician brief advice versus a motivational interview. J Cogn Behav Psychother. 2009; 9(1):1-19. [PMID:20694059]

41. Christensen H, Griffiths KM, Farrer L. Adherence in internet interventions for anxiety and depression. J Med Internet Res. 2009;11(2):e13. [PMID:19403466]

http://dx.doi.org/10.2196/jmir.1194

42. Garber J, Clarke GN, Weersing VR, Beardslee WR, Brent DA, Gladstone TR, DeBar LL, Lynch FL, D’Angelo E, Hollon SD, Shamseddeen W, Iyengar S. Prevention of depression in at-risk adolescents: a randomized controlled trial. JAMA. 2009;301(21):2215-24. [PMID:19491183] http://dx.doi.org/10.1001/jama.2009.788

43. Asarnow JR, Jaycox LH, Duan N, LaBorde AP, Rea MM, Murray P, Anderson M, Landon C, Tang L, Wells KB. Effectiveness of a quality improvement intervention for adolescent 
depression in primary care clinics: a randomized controlled trial. JAMA. 2005;293(3):311-19. [PMID:15657324]

http://dx.doi.org/10.1001/jama.293.3.311

44. Smit F, Willemse G, Koopmanschap M, Onrust S, Cuijpers P, Beekman A. Cost-effectiveness of preventing depression in primary care patients: randomised trial. Br J Psychiatry. 2006;188:330-36. [PMID:16582059]

http://dx.doi.org/10.1192/bjp.188.4.330

45. Evers KE, Cummins CO, Prochaska JO, Prochaska JM. Online health behavior and disease management programs: are we ready for them? Are they ready for us? J Med Internet Res. 2005;7(3):e27. [PMID:15998618]

http://dx.doi.org/10.2196/jmir.7.3.e27

46. Verheijden MW, Jans MP, Hildebrandt VH, Hopman-Rock M. Rates and determinants of repeated participation in a web-based behavior change program for healthy body weight and healthy lifestyle. J Med Internet Res. 2007; 9(1):e1. [PMID:17478410] http://dx.doi.org/10.2196/jmir.9.1.e1

47. Andersson G, Bergström J, Holländare F, Carlbring P, Kaldo V, Ekselius L. Internet-based self-help for depression: randomised controlled trial. Br J Psychiatry. 2005; 187:456-61. [PMID:16260822]

http://dx.doi.org/10.1192/bjp.187.5.456

48. Cuijpers P, Marks IM, van Straten A, Cavanagh K, Gega L, Andersson G. Computer-aided psychotherapy for anxiety disorders: a meta-analytic review. Cogn Behav Ther. 2009; 38(2):66-82. [PMID:20183688] http://dx.doi.org/10.1080/16506070802694776

49. Calear AL, Christensen H, Mackinnon A, Griffiths KM, O’Kearney R. The YouthMood Project: a cluster randomized controlled trial of an online cognitive behavioral program with adolescents. J Consult Clin Psychol. 2009;77(6): 1021-32. [PMID:19968379] http://dx.doi.org/10.1037/a0017391

50. Litz BT, Engel CC, Bryant RA, Papa A. A randomized, controlled proof-of-concept trial of an Internet-based, therapistassisted self-management treatment for posttraumatic stress disorder. Am J Psychiatry. 2007;164(11):1676-83. [PMID:17974932] http://dx.doi.org/10.1176/appi.ajp.2007.06122057

51. Lange A, van de Ven JP, Schrieken B. Interapy: treatment of post-traumatic stress via the internet. Cogn Behav Ther.
2003;32(3):110-24. [PMID:16291543]

http://dx.doi.org/10.1080/16506070302317

52. Van Straten A, Cuijpers P, Smits N. Effectiveness of a webbased self-help intervention for symptoms of depression, anxiety, and stress: randomized controlled trial. J Med Internet Res. 2008;10(1):e7. [PMID:18364344]

http://dx.doi.org/10.2196/jmir.954

53. Borden W. Contemporary psychodynamic theory and practice. Chicago (IL): Lyceum Books; 2009.

54. Griffiths KM, Christensen H, Jorm AF, Evans K, Groves C. Effect of web-based depression literacy and cognitivebehavioural therapy interventions on stigmatising attitudes to depression: randomised controlled trial. Br J Psychiatry. 2004;185:342-49. [PMID:15458995]

http://dx.doi.org/10.1192/bjp.185.4.342

55. Veroff J, Kulka RA, Douvan E. Mental health seeking in America: Patterns of help-seeking from 1957 to 1976. New York (NY): Basic Books; 1981.

56. Corrigan PW, Penn DL. Lessons from social psychology on discrediting psychiatric stigma. Am Psychol. 1999;54(9): 765-76. [PMID:10510666] http://dx.doi.org/10.1037/0003-066X.54.9.765

57. Van Voorhees BW, Watson N, Bridges JF, Fogel J, Galas J, Kramer C, Connery M, McGill A, Marko M, Cardenas A, Landsback J, Dmochowska K, Kuwabara SA, Ellis J, Prochaska M, Bell C. Development and pilot study of a marketing strategy for primary care/Internet-based depression prevention intervention for adolescents (the CATCH-IT Intervention). Prim Care Companion J Clin Psychiatry. 2010; 12(3). [PMID:20944776]

Submitted for publication June 30, 2011. Accepted in revised form February 1, 2012.

This article and any supplementary material should be cited as follows:

Van Voorhees BW, Gollan J, Fogel J. Pilot study of Internetbased early intervention for combat-related mental distress. J Rehabil Res Dev. 2012;49(8):1175-90.

http://dx.doi.org/10.1682/JRRD.2011.05.0095

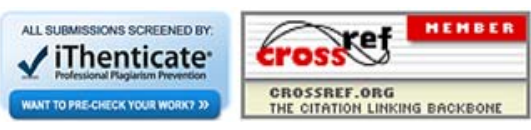

